\title{
Les soubassements religieux d'une réussite éditoriale : le cas de la maison Mame à Tours
}

De la bibliothèque de «bons livres » aux collections pour la jeunesse chrétienne

The religious base of a publishing success: the case of the Mame House in Tours.

From the "Good Books Library" to the eries for Christian Youth

\section{Marie-Pierre Litaudon}

\section{(2) OpenEdition}

Journals

\section{Édition électronique}

URL : http://journals.openedition.org/abpo/2012

DOI : $10.4000 / a b p o .2012$

ISSN : $2108-6443$

\section{Éditeur}

Presses universitaires de Rennes

\section{Édition imprimée}

Date de publication : 30 mai 2011

Pagination : 135-159

ISBN : 978-2-7535-1691-5

ISSN : 0399-0826

\section{Référence électronique}

Marie-Pierre Litaudon, «Les soubassements religieux d'une réussite éditoriale : le cas de la maison Mame à Tours », Annales de Bretagne et des Pays de l'Ouest [En ligne], 118-2 | 2011, mis en ligne le 30 juin 2013, consulté le 01 mai 2019. URL : http://journals.openedition.org/abpo/2012 ; DOI : 10.4000/ abpo. 2012 


\title{
Les soubassements religieux d'une réussite éditoriale : le cas de la maison Mame à Tours
}

\author{
De la bibliothèque de "bons livres " \\ aux collections pour la jeunesse chrétienne
}

\author{
Marie-Pierre LITAUDON \\ Docteur en histoire \\ chercheur associé au CELAM - université Rennes 2 Haute-Bretagne
}

C'est en 1781 que Charles Pierre Mame ${ }^{1}$ reprend à Angers une maison d'édition dont ses enfants développèrent les ambitions à Paris et à Tours. Le destin de la maison établie à Tours par Amand Augustin Mame (17761848) est remarquable. Lorsque ce dernier se retire des affaires en 1833, il est parvenu à ajouter à sa position d'imprimeur du roi (obtenue en 1815) celle d'imprimeur de l'archevêché (1821) et à racheter les brevets et le matériel de ses concurrents. À sa suite, son neveu et associé Ernest Mame garde la charge de l'imprimerie tandis que son fils aîné Alfred hérite de la reliure et de la librairie; tous deux restent associés jusqu'en 1845, date à laquelle Alfred reprend seul l'entreprise. En deux décennies Alfred Mame (1811-1893), doué d'un réel génie des affaires, va mettre sur pied ce qui devint sous Napoléon III un empire de l'édition de masse catholique ${ }^{2}$.

Sans sous-estimer les talents d'entrepreneur d'Alfred Mame, cette réussite n'aurait pas été aussi fulgurante si elle n'avait trouvé dès ses débuts l'appui du clergé tourangeau, et notamment celui du jeune et dynamique

1. Cf. GRILLE, François Joseph, « Mame », Le Bric-à-brac. Paris, Ledoyen, 1853, t. 1, p. 174 179; LETORDU, Jérôme, « L'imprimeur Charles Pierre Mame (1747-1825) ou l'ascension d'un opportuniste angevin ", Recherches vendéennes, ${ }^{\circ}$ 8, 2001, p. 109-132. Charles Pierre Mame, nommé imprimeur le 9 avril 1781, s'installe en reprenant l'atelier de Charles François Billault qui a démissionné en sa faveur (cf. DAUDIER, Matthieu, Imprimerie et librairie à Angers dans la seconde moitié du XVIII siècle (1752-1789), mémoire de maîtrise d'histoire (sous la direction de J. Maillard), université d'Angers, 1998, p. 45).

2. CoRnut, Étienne, "Alfred Mame l'industriel ", Études religieuses, philosophiques, historiques et littéraires, 1894, t. 61, p. 121-147; Du SAussoIs, Auguste, Alfred Mame, célèbre imprimeur et éditeur-libraire français, Lille, Impr. de l'orphelinat de Don Bosco, 1898. 
abbé Dufêtre (1796-1860), vicaire général du diocèse de Tours depuis la fin de 1824 et futur évêque de Nevers ${ }^{3}$. Selon son biographe, l'abbé Dufêtre avait conçu le projet :

" [...] de donner à cette branche si négligée de la librairie [d'éducation] une impulsion toute à l'avantage des bonnes doctrines, et s'en ouvrit à MM. Mame, leur demandant de seconder ses vues, leur promettant son concours le plus actif, s'engageant [...] à recommander partout leurs publications, s'ils consentaient à détruire un certain nombre de livres édités par leurs prédécesseurs, [...] et à ne publier à l'avenir que des volumes [...] approuvés par $\mathrm{M}^{\mathrm{gr}}$ l'archevêque de Tours. Ces propositions furent accueillies avec un généreux empressement ${ }^{4}$."

Devenu évêque, l'abbé Dufêtre « ne pouvait retirer son patronage à l'œuvre dont il avait été l'inspirateur; il le lui conserva [...] jusqu'à son dernier jour ${ }^{5}$ ". À l'appui de ses affirmations, son biographe cite une lettre d'Alfred Mame, en date de 1865 : «M. Dufêtre voulut bien nous diriger dans la publication de nos livres d'éducation; il nous rendit alors un très grand service en organisant lui-même notre Bibliothèque de la jeunesse, en nous cherchant des auteurs, en examinant les manuscrits ${ }^{6}$. " L'entreprise s'inscrit dans le vaste programme de reconquête des âmes, prôné entre autres par $\mathrm{M}^{\mathrm{gr}}$ de Boulogne, archevêque de Troyes de 1808 à 1825, et par $M^{\text {gr }}$ Frayssinous, évêque in partibus d'Hermopolis, grand maître de l'Université, puis ministre de l'Instruction publique et des Affaires ecclésiastiques sous la Restauration. Ce clergé ultramontain invite à la lutte armée contre les mauvaises doctrines ${ }^{7}$. Le livre étant le fer de lance de la modernité libérale, il faut, comme le dira plus tard Dufêtre, " agir par la presse contre la presse, combattre la propagation de l'erreur par la propagation de la vérité ${ }^{8}$ ». La formule est un leitmotiv que l'on retrouve sous diverses formes, tant dans les avant-propos de catalogues que dans les conférences, mandements et instructions d'évêques. Une telle campagne suscite

3. L'implication de l'abbé Dufêtre dans la réussite éditoriale d'Alfred Mame a été mise en évidence par Michel Manson dans sa communication "Mame et Friedel, une rencontre aux origines de la spécialisation de l'éditeur de Tours dans la littérature de jeunesse? » (Journée d'étude du 13 janvier 2009, programme ANR " la Maison Mame à Tours "). Il cite à l'appui de son propos une notice biographique sur Dufêtre réalisée par Marius Guérin dans l'édition critique qu'il établissait des Pamphlets de Claude Tillier, adversaire déclaré de notre abbé. Explorant les implications de cette découverte, j'ai présenté lors de la journée d'étude du 15 janvier 2010, la communication «Mame, l'abbé Dufêtre et les bibliothèques de bons livres. Contexte de création et contraintes éditoriales ", dont le présent article est le prolongement. Je remercie Michel Laurencin, responsable adjoint des archives diocésaines de Tours, pour l'aide qu'il m'a apportée dans mes recherches.

4. Crosnier, Augustin Joseph, Vie de $M^{g r}$ Dufêtre, évêque de Nevers, Paris, Tolra et Haton, 1868, p. 164.

5. Cette remarque explique pourquoi, de sa prise de fonction à Nevers en 1843 à son décès en 1860, la quasi-totalité des ouvrages publiés par la maison tourangelle fut approuvée par l'évêque de Nevers et non par l'archevêque de Tours.

6. Crosnier, Augustin Joseph, Vie de $M^{g r}$ Dufêtre..., op. cit., p. 165.

7. Voir les textes rassemblés sous le titre Considérations sur la propagation des mauvaises doctrines, Paris, Société catholique des bons livres, 1827.

8. Euvre des bons livres de Nevers. L.-M. Fay, 1843, p. 3. (Bib. mun. de Nevers, 3 N 1774). 
en France à partir des années 1820 la création des premières bibliothèques chrétiennes de prêt. Dans leurs travaux respectifs, Claude Savart puis Loïc Artiaga en font un relevé ${ }^{9}$. Mais ils n'offrent pas une description détaillée de celle qui se crée à Tours en 1825 .

Cerner de plus près le projet des " bons livres " de l'abbé Dufêtre, sa maturation (des bibliothèques de prêt aux collections pour la jeunesse), ses modalités de fonctionnement et d'influence, ainsi que la manière dont celles-ci servirent l'ambition d'Alfred Mame, n'est pas chose aisée tant les preuves directes font défaut. Néanmoins, en nous appuyant sur un faisceau d'indices, nous essaierons de montrer que celui-ci s'inscrit, selon toutes probabilités, dans un réseau serré de sociétés aux visées contre-révolutionnaires et apostoliques, associant l'éducation à la lecture de masse. Nous nous attacherons tout d'abord à préciser le contexte de création de la bibliothèque de " bons livres " de Tours. Cette première approche nous amènera à détailler le réseau de personnalités sur lequel elle s'appuie, réseau qui révèle combien ce projet s'inscrit dans une entreprise plus large et plus ancienne, mettant en jeu des sociétés plus ou moins secrètes aux ramifications internationales. Nous reviendrons ensuite sur l'œuvre tourangelle pour voir comment son affiliation à l'oeuvre bordelaise va engager la maison Mame dans la création de collections pour la jeunesse chrétienne que les abbés Dufêtre et Donnet se chargeront de promouvoir.

\section{" Une association pour la propagation des bons livres... "}

Contrairement à de nombreuses œuvres du même type établies en France, l'oeuvre des bons livres de Tours n'a laissé, en l'état actuel de nos connaissances, aucune trace de son existence. Les archives diocésaines et départementales d'Indre-et-Loire, pas plus que la bibliothèque municipale de Tours, ne conservent de mandements ou de correspondances relatifs au sujet. Il n'existe aucun règlement de l'œuvre, aucun compte-rendu d'assemblée générale, pas même un catalogue ou un prospectus ${ }^{10}$. Seul le journal L'Ami de la religion et du roi relate à la date du 15 février 1826 :

"Une association pour la propagation des bons livres s'est formée l'année dernière à Tours; $M$. l'archevêque a encouragé cette œuvre. [...]. Le prélat [en] a confié le soin à l'association formée à Tours, en 1817, par les missionnaires, et à laquelle sont principalement dues les écoles chrétiennes

9. SAVART, Claude, Les Catholiques en France au XIXe siècle : le témoignage du livre religieux, Paris, Beauchesne, 1985. ARTIAGA, Loïc, Des torrents de papier. Catholicisme et lectures populaires au XIX ${ }^{e}$ siècle. Presses universitaires de Limoges, 2007.

10. Je n'ai cependant pas exploré les registres de l'Cuvre bordelaise conservés aux archives diocésaines de Bordeaux, ni la correspondance de l'abbé Donnet (Arch. nat. : Archives privées, 160 AP/1). Par ailleurs, le Journal d'Indre-et-Loire, susceptible d'avoir relaté les modalités de création et de fonctionnement de l'Euvre tourangelle, est actuellement en cours de traitement à la bibliothèque municipale de Tours, de même que le fonds des bibliothèques des grand et petit séminaires, récemment versé par les archives diocésaines. 
aujourd'hui dotées par la ville et encouragées par le conseil général, de manière à offrir une éducation chrétienne à 600 enfans. Une direction des bons livres est formée à l'instar de la société catholique à Paris ; cette direction est composée de cinq membres, dont trois ecclésiastiques; de plus, il $\mathrm{y}$ a une commission de vingt-quatre membres qui travaillent à multiplier les souscriptions et à répandre les bons livres. On a établi à Tours un dépôt central, rue de la Monnaie; là on donne, échange ou prête les livres sur un bon des curés ou autres ecclésiastiques membres de la commission. La bibliothèque a commencé le 24 avril de l'année dernière. Elle s'est formée, dès l'origine, d'environ 2000 volumes donnés par des personnes zélées. Le catalogue est assez varié et renferme plus de 600 articles; les livres les plus usuels y sont au nombre de plusieurs exemplaires. La direction [...] se félicite du succès de ses travaux. Elle réunit aujourd'hui 2500 volumes; le nombre des lecteurs s'est accru, et environ 7 à 800 personnes recueillent les fruits de cet établissement. On se propose de former de petites bibliothèques dans les autres villes ou bourgs du département. M. l'archevêque vient de s'adresser au saint Père pour obtenir des grâces spirituelles en faveur des bienfaiteurs de l'oeuvre."

Ce compte-rendu appelle deux commentaires. Le premier est d'ordre spéculatif. La création de cette bibliothèque intervient quelques mois après la nomination de l'abbé Dufêtre au poste de grand vicaire. Elle pourrait donc être le fruit de ses directives, pour des raisons liées à ses fonctions initiales dans le diocèse de Tours. Depuis 1823 en effet, Dominique Dufêtre officie aux côtés de son plus proche ami qu'il a demandé à rejoindre, l'abbé Ferdinand Donnet (1795-1882), appelé un an plus tôt par l'archevêque de Tours pour diriger les missionnaires de Saint-Martin, congrégation que le prélat vient de fonder pour évangéliser la Touraine.

Tous deux ont été formés au séminaire Saint-Irénée de Lyon, véritable pépinière d'orateurs, de prédicateurs et d'évêques, dirigée par des sulpiciens et placée sous la protection du cardinal Fesch. Ce prélat souhaitait former dans l'ancien couvent des chartreux une congrégation de prêtres voués à l'étude et à la prédication des missions intérieures. Animés d'une même ferveur, les séminaristes Dominique Dufêtre, Ferdinand Donnet et Louis Querbes (futur fondateur des clercs de Saint-Viateur) forment alors un trio si soudé que leurs camarades auraient eu coutume de dire : "Querbes, Dufêtre, Donnet/Trois têtes sous un même bonnet ${ }^{11}$. " Cette amitié trouve sans doute un point d'ancrage dans leur participation à l'une des quatre petites sociétés qu'abrite le séminaire : à côté de la "Pensée pieuse ", des " Mariistes ", des " Amis du cordon ", notre trio ferait partie d'un réseau d'aspirants jésuites ${ }^{12}$. Liés par des vœux de perfection sacerdotale, ils conçoivent leur mission en soldats de Dieu.

11. RoBert, Pierre, Vie du père Louis Querbes, Bruxelles, A. Dervit, 1922, p. 34.

12. Bonnafous, Robert, Un fondateur contrarié. Louis Querbes 1793-1859, Les Clercs de Saint-Viateur, Vourles, 2004-2005, t. 1 « Les années de formation et de fondation », p. 70-75. Voir aussi RoBerT, Pierre, Vie du père... op. cit., p. 36 : "Sans être jésuites, [les Pères de la foi présents dans le diocèse de Lyon] [...] appelaient de tous leurs vœux le rétablissement de la Compagnie de Jésus. Louis Querbes et un petit groupe de séminaristes 
Il n'est pas surprenant, dès lors qu'entre 1823 et 1826 , sous la houlette de Dufêtre et Donnet, les déplacements des missionnaires de Saint-Martin soient l'occasion d'une double croisade, l'une vouant les "mauvais livres ${ }^{13}$ " à l'autodafé, l'autre oeuvrant à la création de bibliothèques chrétiennes, comme à Blois, Vendôme et Romorantin. La bibliothèque municipale de Blois conserve les statuts de celle qui s'établit dans sa ville sous la direction de $\mathrm{M}^{\mathrm{gr}}$ de Sauzin, à la suite de la mission de $1824^{14}$; on y trouve la liste des membres figurant dans son conseil de direction : figures politiques (préfet, maire, député), notaire et juge, nombreuses personnalités religieuses, parmi lesquelles deux vicaires généraux (l'abbé Fabre Des Essarts, futur évêque de Blois, et l'abbé Donnet, supérieur des missions du diocèse). Sur ce modèle nous pouvons émettre l'hypothèse que, devenu le bras droit du nouvel archevêque de Tours, l'abbé Dufêtre avait intégré le conseil de direction de l'Euvre tourangelle.

La maison Mame s'est-elle associée à cette œuvre? L'absence de documents ne permet pas de l'affirmer. Nous ne disposons que d'indices. On sait qu'Amand Mame a su adroitement se rapprocher des cercles catholiques. Dès 1818, l'imprimeur entend ne publier que des "bons livres ". Sa production est dominée dans les années qui suivent par des ouvrages scolaires; s'y ajoute à partir de 1823 un nombre croissant de titres caractéristiques des bibliothèques de bons livres. Imprimeur de l'archevêché depuis février 1821, l'entreprise est bien placée pour répondre à de telles commandes. Et ce d'autant plus que les maisons Mame d'Angers et de Tours figurent parmi la liste des neuf imprimeurs-libraires associés fournie par le Manuel de l'Euvre des bons livres de Bordeaux en $1827^{15}$. Or cette Euvre fait figure d'association-mère et diffuse son modèle auprès d'autres évêchés. Son manuel précise :

"L'EEuvre n'édite point à son propre compte [...] cependant des arrangements ont été pris, pour que les ouvrages soient cédés à l'Euvre à un prix extrêmement modique. [...] Nos libraires seront toujours assortis des ouvrages et éditions dont il sera mention sur le catalogue approuvé par le bureau d'examen. "

partageaient leurs espérances. Pour les fortifier, pour s'encourager mutuellement, ils avaient formé entre eux une petite "Société ", avec réunions et entretiens intimes durant l'année, échanges de lettres pendant les vacances".

13. Sous ce terme générique sont rassemblés les oeuvres des philosophes du xvIII ${ }^{\mathrm{e}}$ siècle (principalement Voltaire et Rousseau), les romans à la mode jugés obscènes et les opuscules protestants colportés par les sociétés bibliques.

14. Association pour l'établissement et l'entretien d'une bibliothèque de bons livres dans la ville de Blois, Impr. de Madame veuve Jahyer, 1826. (Bib. mun. de Blois, LB 4859 et LB 2026).

15. Les autres imprimeurs cités sont : MM. Rusand à Lyon et à Paris, Gauthier à Besançon et à Paris, Méquignon à Paris, Périsse à Lyon et à Paris, Séguin à Avignon, Lavignac à Bordeaux, Mellinet à Nantes et Barbou à Limoges. Euvre des bons livres fondée par $\mathrm{M}^{\mathrm{gr}} \mathrm{d}$ 'Aviau, archevêque de Bordeaux, approuvée par Sa Sainteté Léon XII, adoptée par un grand nombre d'évêques de France. Manuel de l'Euvre. Nantes, impr. Mellinet-Mallassis, 1827. (BnF, D-46387). 
Peut-on aller plus loin? Amand Mame puis Alfred Mame ont-ils pris part au bureau de direction de l'Euvre tourangelle? L'hypothèse n'est pas à exclure, d'autant qu'elle est avérée dans le cas de l'éditeur Barbou. Cet imprimeur associé de l'Euvre bordelaise figure en effet parmi les membres du conseil administratif de l'Euvre de Limoges, au moins à la date de $1849^{16}$. Toujours à l'appui de cette hypothèse, il faut rappeler le nombre important d'ouvrages qui composent la bibliothèque de Tours dès son ouverture. En tant qu'imprimeur associé, Amand Mame avait intérêt à intégrer le conseil de direction de l'association tourangelle. Pour ce faire, quoi de plus obligeant que de s'en montrer le généreux bienfaiteur, en étant du nombre de ces " personnes zélées " ayant offert 2000 volumes? La remarque vaut également pour l'imprimeur Mellinet-Mallassis à Nantes, quand on sait que l'Euvre des bons livres de Nantes dispose dès l'origine d'un fonds de 4000 volumes ${ }^{17}$. En 1825, de tels chiffres placent ces deux bibliothèques chrétiennes parmi les plus importantes de France. L'Ami de la religion et $d u$ roi en relève trois autres. La plus ancienne est la "Bibliothèque chrétienne " de Grenoble, créée en 1818. Elle compte d'emblée 2000 volumes et atteint les 7000 en $1825^{18}$. Celle de Bordeaux débute officiellement en 1820; mais une première expérimentation avait été réalisée par l'abbé Barrault dès 1812, sous forme de bibliothèque personnelle. L'abbé, ayant mûri ses pratiques durant 8 ans, soumit à $M^{\text {gr }}$ d'Aviau un projet de bibliothèques de prêt que ce dernier s'empressa d'approuver. Les premières souscriptions permirent de constituer un fonds de 6820 volumes, passé à 14000 en $1827^{19}$, puis à 50000 en $1836^{20}$. Enfin, la "Société catholique des bons livres ", fondée à Paris en $1824^{21}$, est une association huppée et médiatique, présidée par le duc Matthieu de Montmorency. Elle utilise la presse pour faire connaître en France ses programmes et se vante en 1825 d'avoir créé 125 directions provinciales. Son fonctionnement est en réalité différent des Euvres de bons livres provinciaux, puisqu'elle s'appuie sur un système de parrainage : les riches familles qui souscrivent distribuent dans les foyers populaires des livres édités par la société. Peu adaptée aux réalités pratiques, mais aussi trop ouvertement associée à la sulfureuse Congrégation, cette société disparaît en 1830. D'autres Euvres cependant voient le jour dans les années 1830, sans égaler l'importance de celles d'Angers et de Tours. À titre d'exemple, la Bibliothèque catholique de Cambrai, créée en 1834, ne compte que 300 volumes la première année. Celle qui se forme à Aix en 1836 n'en compte que 400, celle de Toulon 1200.

16. RouSIER, L. (Abbé), Rapport sur l'oeuvre des bons livres. Limoges, impr. Barbou Frères, 1849 (Bib. mun. de Limoges, MAG. P LIM B1587).

17. Catalogue de la Bibliothèque de l'Euvre des bons livres de Nantes, op. cit.

18. L'Ami de la religion et du roi, vol. 45.

19. Ibid., vol. 52 .

20. Pour un historique de l'Euvre, voir l'introduction de Noë RICHTER aux Manuels de l'Éuvre des bons livres de Bordeaux (1834). Réimpression en fac-simile des deux manuels publiés en 1834, l'un à l'attention des associés (I), l'autre à l'attention des directeurs, examinateurs et bibliothécaires (II), Bassac, Plein Chant, 1996.

21. L'Ami de la religion et du roi, t. 40, plusieurs articles. 
Ces premiers éléments posés, l'article consacré à la création de l'Euvre tourangelle appelle un second commentaire. D'après L'Ami de la religion et du roi, l'EEuvre de Tours aurait été fondée sur le modèle parisien de la "Société catholique des bons livres "; mais la description qu'il donne de la société tourangelle montre que son véritable modèle est celui de " l'Euvre des bons livres de Bordeaux ", plus discret mais plus efficace. Son innovation majeure tient à la création d'un réseau hiérarchisé de bibliothèques de dépôt partiel rattachées à une bibliothèque centrale. Ces bibliothèques sont circulantes, c'est-à-dire qu'elles pratiquent le prêt de manière intercommunale. On retrouve également les statuts de l'Euvre bordelaise dans l'organisation de l'association tourangelle en deux bureaux : l'un, restreint, est chargé d'examiner les livres qui doivent entrer dans le dépôt ${ }^{22}$ tandis que le second, plus large, travaille à étendre l'Euvre et conseille le directeur dans ses choix grâce à sa connaissance du terrain.

Si les abbés Donnet et Dufêtre n'ont peut-être pas initié ce modèle à Tours, ils s'en sont montrés les zélés promoteurs au cours de leurs missions. Leur action obéit aux recommandations bordelaises tant par la destruction des mauvais livres et la multiplication des bons (bureaux d'examen, bibliothèques) que par l'attention particulière portée à l'éducation de la jeunesse. Le Manuel de Bordeaux (1827) recommande en effet le développement des dépôts " de correspondance " dans les établissements disposant d'une bibliothèque, notamment scolaire. Celui-ci s'en explique :

" Il est très-important que les jeunes gens se procurent ou du moins apprennent à connaître ces sortes de livres pendant le cours de leurs études; car l'expérience prouve que la plupart au sortir des maisons d'éducation, ne pensent plus aux ouvrages de religion, et ne composent leur bibliothèque que d'ouvrages de littérature, sans aucune distinction des bonnes et des mauvaises éditions. C'est donc un grand service que de les diriger dans ce choix, tandis qu'ils sont sous la conduite de maîtres sages et éclairés. "

Concernant l'examen des livres, il souligne :

«Les livres reconnus susceptibles de correction, retranchement ou modification, sont épurés par les soins du bureau, et réimprimés avec les corrections nécessaires par les imprimeurs de l'Euvre. Les livres classiques et les ouvrages de littérature sont l'objet principal de ce travail. "

Le Manuel rappelle à cette occasion que les directeurs et professeurs des deux séminaires font impérativement partie du bureau d'examen de l'Euvre. C'est ce que l'on observe en effet à Blois, où Ferdinand Donnet cumule en 1825 les fonctions de directeur des séminaires et de membre de la direction de l'Euvre des bons livres.

22. Le Manuel de Bordeaux (I, p. 13) précise que le directeur de l'Euvre dispose seul de la correspondance et des rapports de gestion. Si Dufêtre fut le directeur de l'Euvre de Tours, voilà qui expliquerait pourquoi aucun des registres n'a été conservé dans ce diocèse. Les dossiers l'auront suivi à Nevers, avant d'être détruits à sa mort (il n'en subsiste aucune trace dans les archives diocésaines de Nevers). La direction de l'Euvre revint ensuite à l'archevêché de Tours. 
Résultat de ces travaux, figure enfin dans le Manuel de 1827 une liste d'ouvrages scolaires épurés : des classiques latins et grecs, un cours complet d'histoire, un dictionnaire de langue française et des ouvrages de littérature et de religion : Introduction à la vie dévote, Magasin des pauvres de $\mathrm{M}^{\text {me }}$ Leprince de Beaumont, Caractères de La Bruyère, Fables de La Fontaine, Télémaque, La Henriade, Euvres de Boileau et de J.-B. Rousseau, etc. Il est possible que les ouvrages scolaires publiés par Amand Mame entre 1821 et 1833 s'inscrivent déjà dans ce réseau de diffusion car leur tirage est élevé, entre 4000 et 10000 exemplaires. Une chose est sûre cependant : Amand Mame est l'imprimeur des livres d'instruction primaire du diocèse de Tours ${ }^{23}$.

Alors que le Manuel de 1827 semblait viser principalement les maisons d'éducation et les collèges, celui de 1834 fait preuve d'une attention accrue aux petites écoles populaires. Les œuvres de bons livres doivent désormais " servir les pauvres, en facilitant aux Frères des écoles chrétiennes et aux bons instituteurs des campagnes, des moyens d'instruction et d'encouragement pour leurs élèves, soit par le prêt de livres, même classiques, soit par les dons de quelques petites récompenses " (I, p. 10).

C'est très exactement ce lien qui s'établit à Tours entre l'établissement d'écoles chrétiennes et la constitution de bibliothèques de bons livres. Selon L'Ami de la religion et du roi, c'est à "l'association formée à Tours, en 1817, par les missionnaires, et à laquelle sont principalement dues les écoles chrétiennes " de la ville qu'a été confiée l'Euvre des bons livres créée en 1825. Cette première association est l'œuvre des Missionnaires de France, compagnie reformée à la Restauration par les pères Rauzan et Forbin-Janson ${ }^{24}$. En 1817, ceux-ci parcourent l'Ouest de la France. Leur apostolat vise notamment à rétablir les frères des écoles chrétiennes afin de lutter contre le développement des écoles mutuelles suspectées dans certains milieux cléricaux de répandre les principes protestants dans les villes et les campagnes. De même, à Nantes, "la venue des Frères est due à une association de souscripteurs formée à la suite de la mission prêchée en 1816. Elle prend le nom de "Société de la Providence" et se place sous la direction de l'abbé Bodinier ${ }^{25}$ ". Or, celui-ci n'est autre que le futur directeur de l'EEuvre des bons livres de Nantes. Là comme à Tours, l'association responsable de l'ouverture d'écoles est aussi celle qui gère les dépôts de bons livres ${ }^{26}$. Tout porte à croire que la double responsabilité de ces

23. La circulaire du 10 décembre 1824 aux curés du diocèse donne une liste de 13 ouvrages dont 11 sont disponibles chez M. Mame et 2 chez les frères des écoles chrétiennes, place de la Riche. Arch. dioc. de Tours, cote 1611.

24. La congrégation des Missions de France, créée par le cardinal Fesch en 1808, avait été dissoute par Napoléon $1^{\mathrm{er}}$ en 1811.

25. Chalopin, Michel, L'Enseignement mutuel en Bretagne de 1815 à 1850, thèse de doctorat en histoire contemporaine, Université Rennes II, 2008, p. 184.

26. Le Catalogue de la Bibliothèque l'Euvre des bons livres de Nantes (1825) souligne à ce propos que des "dépôts ont été confiés aux directeurs des écoles chrétiennes de cette ville". 
associations obéit à un projet mené sous le sceau du secret par un réseau de personnalités religieuses, à la fois amical et militant, qu'il nous faut à présent envisager.

\section{Réseau(x) secret(s) et apostolat par le livre}

L'association créée à Tours à la suite de la mission de 1817 connaît, suivant les archives, deux appellations. Nommée "Société pour la défense de la religion catholique ", d'après l'historique de l'école Notre-Dame-laRiche $^{27}$, elle devient "Association de la mission " dans les archives diocésaines qui conservent le dossier de l'abbé Guespin, curé de Notre-Damela-Riche ${ }^{28}$. Ce prêtre réfractaire, exilé à Rome en 1792, fut l'administrateur secret du diocèse de Tours sous la Terreur et un proche correspondant du père Clorivière, au point de devenir un membre actif de sa nouvelle Société du Cœur de Jésus.

Dès son retour à Tours en 1803, l'abbé Guespin installe les carmélites au couvent Notre-Dame-la-Riche, les déclare enseignantes et il demande à Clorivière et à mademoiselle de Cicé de lui fournir quelques filles du Cœur de Marie, société sœur de la première, pour aider les carmélites dans leurs fonctions. En 1818, lorsqu'il se joint à l'Association de la mission, c'est encore lui qui œuvre à l'installation des frères des écoles chrétiennes, tant par ses démarches épistolaires auprès de la maison mère à Lyon que par ses dons financiers. Mais il n'est pas seul à agir. Le vicaire général Nicolas Simon, chanoine et curé de Saint-Martin (que Balzac immortalisa dans Le Curé de Tours) est lui aussi une figure très active du clergé tourangeau sous l'Empire et la Restauration. Connu pour avoir voué sa fortune à la cause des pauvres, il œuvre aux côtés de l'abbé Guespin pour établir de petites écoles chrétiennes dans la ville. En 1817, il héberge à ses frais les Missionnaires de France venus évangéliser la Touraine. À partir de 1819, il règle et finance l'installation des sœurs et des frères des Sacrés-Cœurs de Jésus et de Marie (picpuciens), les premières chargées des petites écoles de filles, les seconds du petit et du grand séminaires de Tours. Cette congrégation est dirigée par le père Coudrin et la mère Henriette Aymer de la Chevalerie. Cette dernière n'est autre que la nièce de $\mathrm{M}^{\mathrm{gr}} \mathrm{Du}$ Chilleau, archevêque de Tours (1818-1824) ${ }^{29}$ et protecteur du projet scolaire de Nicolas Simon. Le père Coudrin est un ancien séminariste de Poitiers que Charles d'Aviau ${ }^{30}$, alors

27. Cette institution, dont le développement sous la Restauration est redevable à cette association, semble disposer d'archives de premier ordre, dont les registres de cette fameuse association. Mais sa direction décline toute demande de consultation et refuse de verser son fonds aux archives diocésaines.

28. Arch. dioc. de Tours, Dossier «Abbé Jean-François Guespin (1745-1821)", 1114.

29. Issu de la haute noblesse poitevine, Jean-Baptiste du Chilleau fut l'aumônier de la reine avant de faire partie du clergé réfractaire. Il rentre en France en 1815, après avoir successivement habité la Suisse, la Bavière et l'Autriche. Il fut hébergé jusqu'à sa nomination à Tours par les picpuciens.

30. Charles François d'Aviau du Bois de Sanzay (1736-1826), issu de la vieille noblesse poitevine, est nommé chanoine de la collégiale Saint-Hilaire de Poitiers, puis grand vicaire 
grand vicaire du diocèse, prend sous son patronage et fait entrer en 1785 dans l'Associatio amicorum (Aa) de la ville ${ }^{31}$. S'il contribue à la promotion du jeune Coudrin, d'Aviau est aussi l'ami de Nicolas Simon. Tous deux ont fait leurs études au collège jésuite de La Flèche, puis au séminaire d'Angers. Durant la période prérévolutionnaire, lorsque le chanoine d'Aviau se rend à Tours pour se livrer à des prédications, il se fait héberger par l'abbé Simon. Ses talents lui valent d'être nommé vicaire général honoraire du diocèse de Tours. Son réseau d'influence ne s'arrête pas là. Un des deux directeurs des Missions de France, le père Rauzan (1757-1847), dont nous avons eu l'occasion de parler, n'est autre que l'ancien vicaire général de $\mathbf{M}^{\mathrm{gr}} \mathrm{d}$ 'Aviau, nommé par celui-ci en 1802, lorsqu'il prit ses fonctions à Bordeaux. Il ne quitte $\mathrm{M}^{\mathrm{gr}}$ d'Aviau qu'en 1808, pour rejoindre le cardinal Fesch et le diocèse de Lyon. Le prélat souhaitait placer Rauzan à la tête du séminaire SaintIrénée, véritable pôle des hautes études religieuses. $\mathrm{M}^{\mathrm{gr}} \mathrm{d}$ 'Aviau ne cède son grand vicaire qu'à regret : " Je vous prête un temps à son Éminence le cardinal archevêque de Lyon; mais souvenez-vous que c'est à des conditions avantageuses : il faudra qu'au retour de la cession que je lui fais, elle m'envoie un jour une colonie d'ouvriers évangéliques ${ }^{32}$. " Parmi ces " ouvriers évangéliques " figureront quelques années plus tard les prêtres Dufêtre et Donnet. Dès lors, est-ce un hasard si Ferdinand Donnet achève sa formation dans cet établissement, après avoir étudié au collège Sainte-Barbe d'Annonay, fondé par $\mathrm{M}^{\mathrm{gr}} \mathrm{d}^{\prime} \mathrm{Aviau}{ }^{33}$, proche ami de la famille Donnet ${ }^{34}$ ?

L'apparition récurrente de $\mathrm{M}^{\mathrm{gr}} \mathrm{d}$ 'Aviau dans le parcours des principaux acteurs du projet apostolique entrepris à Tours invite à s'interroger sur les soubassements de cette entreprise. Elle semble s'inscrire dans un projet plus vaste mettant en jeu un réseau de sociétés plus ou moins secrètes, aux origines jésuites.

$\mathrm{M}^{\mathrm{gr}} \mathrm{d}$ 'Aviau est une des grandes figures du clergé réfractaire. Dénommé le "saint archevêque " par Pie VI et Pie VII, le prélat fit œuvre de résistance politique moins sur la scène publique que par le biais de sociétés pieuses, d'inspiration jésuite, au titre desquelles figurent d'abord les congrégations mariales. Implantées dans les collèges et placées sous la houlette des maîtres, ces sociétés réunissent les élèves les plus studieux, afin de sus-

du diocèse avant d'obtenir l'archevêché de Vienne. Il s'exile en 1791 mais revient en 1797, déguisé en paysan, pour administrer secrètement ses diocèses. Il est nommé archevêque de Bordeaux en 1802, fonction qu'il occupera jusqu'à sa mort.

31. Waché, Brigitte, "Prêtres exilés pendant la Révolution et germes de renouveau à partir de l'exemple de Guillaume Joseph Chaminade ", Mundo marianista, n 8, 2010, p. 33.

32. Delaporte, Alain, Vie du très-révérend père Jean-Baptiste Rauzan, Paris, Jacques Lecoffre \& $\mathrm{C}^{\mathrm{ie}}, 1857$, p. 50-52.

33. À l'époque où celui-ci administre le diocèse de Viviers (1891-1801). C'est aussi dans ce collège que se forma Fabre des Essarts (futur évêque de Blois), avant de rejoindre le séminaire de Viviers, où $\mathrm{M}^{\mathrm{gr}}$ d'Aviau avait probablement établi une Aa. Cf. LANFREY, André, "AAS et petites sociétés. Les associations de clercs des séminaires sulpiciens de 1770 et 1860 ", Revue d'histoire de l'Église de France, 2007, t. 93, p. 163.

34. Notice biographique de Donnet, Collection intégrale et universelle des orateurs sacrés, Paris, J.-P. Migne, 1858, t. 98. 
citer des vocations sacerdotales par la prière, les lectures spirituelles, la visite des hôpitaux ou des prisons. Dès ses premières années au collège de La Flèche, Charles d'Aviau intégra la communauté des Saint-Anges, tandis que Nicolas Simon, de quelques années son cadet, rejoignit la Congrégation de la Vierge.

Les sociétés mariales fournissent un réservoir de séminaristes parmi lesquels sont ultérieurement recrutés les membres des AAS. Ces " assemblées d'amis " sont unies dans la règle et le secret par un même idéal de perfection voué à l'apostolat ${ }^{35}$. Sans disposer d'une organisation centralisée, les AAs entretiennent des liens interdiocésains qui facilitent le partage d'institutions et l'échange de personnel. Rassemblant l'élite spirituelle, elles pèsent fortement dans la promotion ecclésiale et contribuent au renouvellement de la vie religieuse dans la mesure où des fondateurs de congrégations surgissent de leur sein. Une lettre de M. Tresvaux, supérieur de l'AA de Paris, datée de 1837, déclare à ce propos : "Voilà 3 grandes œuvres dues à l'AA : les Missions Étrangères, les Missions de France et la Congrégation des Sacrés-Cœurs de Jésus et de Marie dont M. Coudrin (de l'Aa de Poitiers) est le supérieur général ${ }^{36}$."

Si le jeune Coudrin doit à Charles d'Aviau d'avoir été remarqué puis introduit dans l'Aa de Poitiers, le saint prélat est lui-même issu de l'AA de Bordeaux. Il entretient des liens étroits avec l'Aa de Lyon, de Chambéry et d'Annecy, sans doute parce qu'elles sont l'oeuvre d'un ancien membre de l'AA de Bordeaux ${ }^{37}$, tout comme l'AA établie à Turin ${ }^{38}$; mais aussi parce que les diocèses de Chambéry et d'Annecy se trouvent à la Révolution rattachés à l'archevêché de Vienne. En 1791, le séminaire d'Annecy fournit à $\mathrm{M}^{\mathrm{gr}}$ d'Aviau sur le chemin de l'exil, un asile sûr avant qu'il ne gagne Turin, puis Rome. Ces villes sont autant d'étapes d'un puissant réseau contrerévolutionnaire et ultramontain, où les Aa françaises se mêlent aux Amicizie italiennes. C'est sur la base de ce réseau que s'organisa sous l'Empire la correspondance clandestine du pape, emprisonné à Savone, et de son épiscopat $^{39}$.

35. Pour un rapide historique des AAS, voir LESTRA, Antoine, Les Secrets du clergé clandestin : Le Père Coudrin, fondateur de Picpus. Paris, Lardanchet, 1952 (première partie, chapitre 2 : «La Société du Cor Unum. L'AA »).

36. Ibidem.

37. Selon les travaux de POUTET, Yves et ROUBERT, Jacqueline, qui ont travaillé sur les Annales de l'AA de Lyon, Bordeaux serait, plus que Toulouse, le centre d'un réseau d'AAS dont Lyon ne serait qu'un relais. Cf. "Les Assemblées secrètes des XVII ${ }^{e}$-XVIII relation avec l'Aa de Lyon ", Divus Thomas, $\mathrm{n}^{\circ}$ 70, 1967.

38. Cf. LANFreY, André, "AAS et petites sociétés... ", op. cit., p. 161; Waché, Brigitte, "Prêtres exilés... ", op. cit., p. 33. En fait, les AAs ne se limitent pas à la France. Comme le souligne Brigitte Waché : " On en trouve trace au Canada, en Italie, et en particulier à Rome, en Bavière et en Suisse. "

39. Cf. BerthiER DE SAUvigny, Guillaume, "Un épisode de la résistance catholique sous le Premier Empire : l'affaire d'Astros ", Revue d'histoire de l'Église de France, t. 35, n 125, 1949, p. 49-58. À Chambéry, l'abbé Rey, fervent disciple et « ami " de $M^{\mathrm{gr}}$ d'Aviau, est du nombre de ces résistants qui se firent agents du duc Mathieu de Montmorency. 
La première Amicizia, ou "Amitié chrétienne ", fut créée à Turin entre 1778 et 1780 par un ancien jésuite, Nicolas de Diessbach. Elle fut donc contemporaine de l'AA turinoise. Les deux associations partagèrent très tôt leurs membres, parmi lesquels Bruno Lanteri, disciple et successeur de Diessbach. Le sceau du secret, tout comme le double but de sanctification personnelle et d'apostolat, appelait ce rapprochement. Mais les Amicizie se distinguaient fondamentalement des AAs françaises, outre par la place faite aux laïcs, par l'importance accordée dans sa stratégie apostolique à la puissance de la presse. Tout le programme de l'Amitié était en effet organisé autour de la propagation systématique des "bons livres ", seule capable de répondre à la corruption et à l'incrédulité semées par les publications des ennemis de l'Église. Jean Guerber résume parfaitement les efforts déployés à cet effet :

" [...] repérage des meilleurs ouvrages et établissement de catalogues, diversifiés en fonction des besoins des lecteurs; constitution de bibliothèques réservées aux adhérents [...] mais aussi de bibliothèques de prêt; encouragement et soutien apportés aux éditeurs, comportant parfois une participation aux frais d'impression; distribution gratuite de livres. On songea aussi à découvrir les talents littéraires ignorés, à former et à lancer de jeunes auteurs, à créer des périodiques; mais ces derniers points, sans doute trop ambitieux, ne furent réalisés qu'avec un succès relatif ${ }^{40}$."

On perçoit à la lecture de ce programme tout ce que les Euvres de bons livres françaises doivent au modèle promu par Diessbach et publié pour la première fois à Turin en 1771 sous le titre Le Chrétien catholique invariablement attaché à sa religion. L'ouvrage fut édité en France en 1826 par la " société catholique des bons livres ", sous une forme abrégée qui ne contient ni le projet de bibliothèque, ni le catalogue de bons livres proposé par l'auteur. Cette publication témoigne néanmoins d'une filiation idéologique, de même que l'ambitieux Catalogue de la Bibliothèque chrétienne de Grenoble (1818) ${ }^{41}$ est l'héritier des catalogues élaborés par les Amicizie... et promus par Joseph de Maistre. En témoigne une lettre qu'il adresse au comte de Stolberg en décembre 1817 : "Il s'est établi à Turin, depuis quelques années, sous le nom modeste d'Amis catholiques, une société dont le but principal est de propager la lecture des bons livres, en les tenant à un prix très bas [...] afin de faire circuler la bonne doctrine jusque dans les dernières veines de l'État ${ }^{42}$. " Après avoir décrit brièvement le contenu du catalogue où dominent les genres " polémique, historique et ascétique ", Joseph de Maistre reprend : " Notre correspondance s'étend déjà à Rome et dans les principales villes d'Italie, à Paris, à Vienne, à Genève, etc. Nous avons pensé, Monsieur le Comte, que vous associeriez volontiers votre respectable nom à ceux dont vous trouverez la liste ci-jointe ". L'Ami de

40. GUERBER, Jean, Le Ralliement du clergé français à la morale liguorienne, Universite gregoriana Editrice, 1973, p. 176.

41. Bib. mun. de Grenoble, O 10301.

42. Euvres complètes de Joseph de Maistre, Lyon, Vitte et Perrusel, 1884-1886, t. 14, p. 116-117. 
la religion et du roi relève pour sa part en 1823, à propos des associations pour la propagation des bons livres en France : "Nous savons qu'il existe à Turin une société de ce genre; elle compte des membres très-distingués, dont l'un nous a fait l'honneur de nous écrire à nous-mêmes pour cet objet, et de solliciter notre coopération à une oeuvre dont il peut résulter tant de bien " (t. 36, p. 151). Si cet article intervient après le décès de Joseph de Maistre, survenu en 1821, la lettre dont il est fait mention peut être antérieure de quelques années.

Aussi, la présence des ouvrages de J. de Maistre ou de ceux de Liguori dans le fonds des premières bibliothèques françaises de bons livres doit être regardée comme un indice supplémentaire de l'influence exercée par le modèle italien, relayé par les Missionnaires de France et les sociétés parisiennes. Dans le prolongement, celles-ci vont ajouter aux livres d'éducation et de piété d'Ancien Régime (redevables pour beaucoup aux jésuites), des ouvrages signés par de grands noms de la Congrégation (Laurentie, de Bonald, Frayssinous, Emery, Legris-Duval ${ }^{43}$ ). Les Catalogues de 1833 et 1836 de l'Euvre de Toulouse témoignent de cette " transmission " et l'on ne s'en étonnera pas. La création de cette bibliothèque est l'oeuvre de $M^{\text {gr }}$ d'Astros (probablement issu de l'Aa d'Aix, puis membre de l'AA de Paris, et finalement de la Congrégation). Mais elle bénéficie aussi du travail mené silencieusement par $\mathrm{M}^{\mathrm{gr}}$ d'Aviau et son bibliothécaire en chef, l'abbé Barrault ${ }^{44}$. S'il ne fait guère de doute que $\mathrm{M}^{\mathrm{gr}} \mathrm{d}$ 'Aviau a rapporté d'Italie le modèle initié par Diessbach, il en a surtout retenu sa structure organique et perfectionné, par le biais de son maître d'oeuvre, l'abbé Barrault, ses modalités de diffusion. À la différence des sociétés parisiennes, qui privilégièrent un apostolat d'élite, l'Euvre bordelaise se montre dès ses débuts soucieuse de toucher le lectorat populaire et la jeunesse.

À cet effet, elle accorde une place grandissante aux formes courtes où l'instruction morale prend les dehors de récits "amusants " et où la fiction romanesque a sa place. La nécessité de s'adapter aux réalités du temps en fournissant aux lecteurs une nourriture chrétienne attrayante, avait d'ailleurs amené $\mathrm{M}^{\mathrm{gr}} \mathrm{d}$ 'Aviau à composer un roman, Mélanie et Lucette ou les avantages de l'éducation religieuse, publié en 1811 à Poitiers chez Barbier. Ce texte initialement destiné aux familles " d'honnête condition ${ }^{45}$ ", deviendra un classique des bibliothèques de bons livres. Le passage de ce

43. Les trois derniers sont d'anciens membres de l'AA de Paris. Cf. LANFREY, André, "AAS et petites sociétés..." " op. cit., p. 159.

44. Pour une notice nécrologique de l'abbé Barrault, réalisé par son bras droit, l'abbé Taillefer, voir L'Ami de la religion, 1839, t. 101, p. 311-316.

45. Le dessein de $\mathrm{M}^{\mathrm{gr}}$ d'Aviau était " de réfuter une à une, par une preuve de fait qui les confond toutes, les maximes funestes que les soi-disant amis des lumières et du mouvement s'efforçaient de substituer aux anciennes dans l'éducation de la jeunesse. Déjà un grand nombre de familles, parmi les plus considérables et les plus considérées de la contrée, avait subi cette influence [...]. Il était alors du devoir du saint prêtre [...] de le signaler et de le combattre ». Lyonnet, Jean-Paul François Marie, Histoire de $M^{g r}$ d'Aviaudu-bois-de-Sanzay, Paris, J. Lecoffre et Cie, 1847, p. 123-124. 
premier public à celui élargi des classes populaires s'explique assez bien par le large panel social mis en scène dans le roman. Y sont en effet opposés deux éducations et deux mondes: l'un, urbain et mondain, cède à la frivolité et découvre dans ses négligences sa propre punition; l'autre, rural et modeste, trouve dans la sage pratique des vertus religieuses la voie de la réussite. L'ouvrage ne figure pourtant pas dans le catalogue de l'Euvre de Bordeaux (1834), peut-être par souci de modestie. Cela dit, l'abbé Lyonnet relate : "Devenu [...] Pontife d'une grande Eglise, M. d'Aviau ne cessa de conseiller à ses diocésains le lecture de ce petit écrit; il engagea même, en pleine retraite pastorale, les ecclésiastiques qui y assistaient, à le répandre autant qu'ils le pouvaient $[\ldots]^{46}$."

Amand Mame fut le premier à rééditer cet ouvrage en $1823^{47}$, avant même la création de la Bibliothèque de bons livres de Tours. Cette publication ne témoigne-t-elle pas d'échanges privilégiés entre Bordeaux et Tours? Vicaire général honoraire de la ville, protecteur des picpuciens placés à la tête des deux séminaires, fidèle ami de Nicolas Simon (décédé en 1822) mais aussi de la famille Donnet, dont le fils Ferdinand vient d'être nommé à la tête des Missionnaires de Saint-Martin, $M^{\text {gr }}$ d'Aviau était bien placé pour diffuser son oeuvre et ses projets par le truchement du clergé tourangeau.

Si les premiers acteurs de ce réseau disparaissent entre 1821 et 1826 , il revient aux abbés Donnet et Dufêtre, et au nouvel archevêque $M^{\text {gr }}$ de Montblanc (jusque-là coadjuteur de $\mathrm{M}^{\mathrm{gr}}$ du Chilleau) d'étendre à Tours l'œuvre entamée par leurs prédécesseurs. Dominique Dufêtre, tout comme son ami Ferdinand Donnet, est une figure de la prédication. L'homme est aussi un excellent organisateur qui multiplie les Euvres, dote les congrégations enseignantes de structures solides, pourvoit à leur formation par l'établissement de séminaires et veille à leur expansion sur le long terme. Dès ses années de grand vicaire, il sait se rendre indispensable à $\mathrm{M}^{\mathrm{gr}} \mathrm{de}$ Montblanc qui lui accorde officiellement sa confiance... et officieusement tous pouvoirs. Le fait que les deux hommes soient membres de la Congrégation depuis 1820 n'est peut-être pas étranger à cette étroite " collaboration ${ }^{48}$ ". Quoi qu'il en soit, l'autorité acquise par l'abbé Dufêtre ne peut qu'avoir servi le projet proposé aux éditeurs Mame en 1833, projet sans doute mûri de longue date aux côtés de Ferdinand Donnet. À cet égard, il serait bon de s'interroger sur le voyage entrepris en 1829 par $\mathrm{M}^{\mathrm{gr}} \mathrm{de}$

46. Ibid., p. 124.

47. MichaUd, Joseph François et MichaUd, Louis Gabriel, Biographie universelle ancienne et moderne. Paris, L.-G. Michaud, 1834, vol. 56, p. 600. L'ouvrage signale que cette nouvelle édition paraît à Paris et à Tours. La Bibliographie de la France ne la mentionne pas et la $\mathrm{BnF}$ ne conserve que la seconde édition parue chez Mame en 1826. La Société catholique des bons livres ne publie le roman qu'en 1829, à la suite de Preuves simples et claires de la divinité de Jésus-Christ.

48. Grandmalson, Geoffroy de, La Congrégation (1801-1830), Paris, Plon, Nourrit \& Cie, 1889, p. 177 et 180. L'auteur dit s'appuyer sur des documents manuscrits. Dufêtre aurait ainsi assisté aux réunions de la rue du Bac, alors même qu'il étudiait au séminaire SaintIrénée de Lyon. 
Montblanc et les abbés Dufêtre et Donnet ${ }^{49}$. Réunis pour une cause que le biographe de Dufêtre ne précise pas, tous trois poussent jusqu'à Rome une excursion dans le Midi, en passant par Chambéry et Turin. Si ce trajet évoque celui des AAS et des Amicizie, les trois hommes ne sont officiellement animés que par le désir de visiter la ville éternelle et d'y recevoir la bénédiction du pape. Ils obtiennent une entrevue durant laquelle Dufêtre s'entretient personnellement avec le Pontife. Mais lorsque ce dernier lui demande s'il a une faveur particulière à lui présenter, Dufêtre n'aurait rien trouvé d'autre à solliciter que " de bénir [ses] missions ", requête que son biographe présente comme un acte de parfaite abnégation... La gratuité de ce voyage paraît pourtant peu probable, compte tenu de la personnalité de Dufêtre et de l'identité de ses compagnons. D'autant que Dufêtre profite aussi de ce séjour pour s'entretenir longuement avec le cardinal Fesch qui réside désormais à Rome. On peut donc se demander si Dufêtre ne vient pas plutôt obtenir la bénédiction d'un projet lié aux "bons livres " et à l'éducation, projet dont il cherche à s'assurer pour l'avenir le soutien au plus haut niveau ${ }^{50}$.

La suite à donner à ce projet n'est soumise à MM. Mame qu'en 1833. La Révolution de 1830 oblige Dominique Dufêtre à se tenir caché en Anjou pendant plus d'un an, avant de pouvoir réintégrer ses fonctions et, progressivement, reprendre sa stratégie apostolique. Ne pourrait-on voir dans les Lettres sur l'Italie (1837), un des tout premiers titres parus dans la "Bibliothèque de la jeunesse chrétienne " de l'éditeur Mame, un hommage rendu par Dufêtre à la bénédiction du Pontife? En effet, ce texte de Dupaty tant " vanté par les littérateurs et les philosophes " et où "l'impiété [était] poussée jusqu'au fanatisme " trouve dans cette édition " revue et purgée avec soin par une société d'ecclésiastiques " une forme où l'on " ne trouvera pas un seul mot qui puisse blesser la religion et la morale, et [que] la jeunesse pourra maintenant lire avec fruit ${ }^{51}$ ". La publication de ce récit de voyage est en outre l'occasion pour Dufêtre d'ajouter en appendice un extrait de son propre voyage à Rome. Si l'édition originale ne mentionne pour auteur que " abbé *** ", les éditions ultérieures précisent " abbé D. ". Or, on trouve mentionnée dans ce récit une entrevue de son auteur avec Pie VIII, le 15 juin... date qui correspond à celle donnée par l'abbé Crosnier pour relater l'entrevue de Dufêtre avec le pape ${ }^{52}$.

Ce tour d'horizon des liens tissés entre les membres des sociétés secrètes permet de mieux comprendre sur quel réseau s'appuie l'entreprise

49. L'abbé Donnet a réintégré le diocèse de Lyon depuis 1827 mais est resté chanoine d'honneur et grand vicaire du diocèse de Tours.

50. Le second voyage à Rome de $\mathrm{M}^{\mathrm{gr}}$ Dufêtre, en 1852, n'est sans doute pas plus désintéressé que le premier. Il vient en effet présenter au Pontife les résultats de son action en faveur d'un retour à la liturgie romaine... dont Alfred Mame se fera l'imprimeur de la première heure. Ces efforts conjugués vaudront à l'éditeur d'obtenir l'exclusivité du rite romain dans le monde entier.

51. Les citations sont extraites de la page de titre et de la notice introductive.

52. Crosnier, Augustin-Joseph, Vie de M Mr Dufêtre..., op. cit., p. 45. 
du vicaire général Dufêtre et comment son action apostolique se construit sous la Restauration, aux côtés de l'abbé Donnet. Reste à préciser en quoi la proposition faite par l'abbé Dufêtre à MM. Mame s'inscrit dans une stratégie réfléchie qui ouvre à l'éditeur Alfred Mame la perspective d'un vaste marché captif favorable à son développement industriel.

\section{Les collections pour la jeunesse de l'éditeur Mame dans le réseau des CEuvres de bons livres}

Le projet soumis par l'abbé Dufêtre aux imprimeurs-libraires Mame en 1833 impose un partage clair des tâches : l'abbé Dufêtre se réserve la direction éditoriale des collections pour la jeunesse qu'il souhaite voir imprimées, avec l'approbation de l'archevêque, tandis MM. Mame se partagent les responsabilités technico-commerciales de l'impression et de la diffusion.

Lorsque la maison Mame s'engage dans cette " coopération ", l'Euvre tourangelle des bons livres vient de s'affilier officiellement à l'Euvre bordelaise ${ }^{53}$, élevée depuis 1831 par Grégoire XVI en archiconfrérie. Désormais, toutes les institutions qui souhaitent s'agréger à la société mère de Bordeaux bénéficieront de ses indulgences et rentreront dans un réseau international de diffusion. Sous le contrôle du Saint-Siège, l'archiconfrérie est en effet chargée de la propagation des bons livres " jusque dans les Missions lointaines ${ }^{54}$ ".

L'archiconfrérie fait circuler dès 1832 un catalogue rassemblant les meilleurs ouvrages examinés par les comités ecclésiastiques de chaque association et dont les rapports remontent annuellement à la maison mère ${ }^{55}$. Il est clair que l'abbé Dufêtre a très bien senti le parti qu'il y avait à tirer de l'archiconfrérie des bons livres. Mais dans cette course éditoriale, les imprimeurs-libraires Mame ne sont pas les premiers. La maison Lefort à Lille les a devancés. L'extrait de catalogue que l'archiconfrérie diffuse en 1834 nous apprend que 66 des 200 ouvrages répertoriés sont édités par cette maison. Lefort est le seul éditeur mentionné et recommandé pour ses prix les moins chers. Les titres si avantageusement présentés sont extraits de sa " Nouvelle Bibliothèque catholique ", créée en janvier 1827. Il n'est pas impossible que comme pour Mame à Tours, cette collection trouve

53. Le rapport de l'Euvre bordelaise de 1833, dont L'Ami de la religion se fait l'écho (t. 76, p. 402), indique que cette Euvre n'a pas tenu de réunion générale en 1831 et 1832 et qu'en 1833 "MM. Barrault et Taillefer proposent aux sociétés des bons livres existant en différentes villes de s'affilier à celle de Bordeaux [...]. Toulouse et Tours viennent de répondre à leur appel ".

54. Manuel de Bordeaux (I, p. 28-29). Avant son accession au pontificat, Grégoire XVI avait déjà la charge de la propagation des livres saints dans le monde.

55. Ce catalogue de 54 pages, publié en 1832, est mentionné par l'abbé Taillefer dans la notice nécrologique qu'il consacre à l'abbé Barrault. $C f$. note 44 . Le catalogue de 200 titres publié avec le Manuel de 1834 n'en est qu'un extrait, destiné à l'établissement de dépôts de campagnes ou de petites villes. 
en partie sa justification dans l'ouverture, fin 1825, d'une bibliothèque de bons livres à Lille, œuvre soutenue par la Société catholique des bons livres ${ }^{56}$. On peut imaginer que cette dernière a aussi apporté son soutien à l'éditeur Lefort, connu pour sa fidélité à l'Église et aux Bourbons. Dès décembre 1826, L'Ami de la religion et du Roi annonce la création prochaine de la "Nouvelle Bibliothèque catholique " et donne la liste des titres de la 1re livraison, comme celle des livraisons suivantes (t. 50, p. 218-219 et 408409 ; t. 52, p. 136). Un tel privilège ne sera pas accordé à la maison Mame. Pour s'assurer de son autorité sur le marché des bibliothèques de bons livres, Lefort s'entoure d'ecclésiastiques éclairés et fait appel à des auteurs prolixes, tel Exauvillez (que l'on retrouvera chez Mame) qui lui fournit en 1831 un petit roman, La Bibliothèque de Saint-Gervais, narrant l'érection d'une bibliothèque paroissiale par un saint curé convaincu de son devoir apostolique. L'ouvrage est un véritable manuel d'apprenti bibliothécaire, fournissant jusqu'aux titres à commander. L'année suivante, en 1832, Lefort publie le Guide du lecteur chrétien pour servir dans le choix des ouvrages et de la formation des bibliothécaires, réalisé à la demande de ses souscripteurs, si l'on en croit l'introduction. Ce catalogue, très nourri, est l'occasion pour l'éditeur de préciser quels sont les titres publiés par sa maison. Au début des années 1830, Lefort dispose donc d'une avance considérable sur ses concurrents.

Néanmoins, l'entreprise Dufêtre/Mame va bénéficier à partir de 1836, année de création de la "Bibliothèque de la jeunesse chrétienne ", d'un atout majeur pour combler son retard. L'abbé Donnet, coadjuteur de l'évêque de Nancy, est promu à l'archevêché de Bordeaux. Il entre en fonction en 1837. Abritant dans son diocèse la société mère de l'archiconfrérie, $\mathrm{M}^{\mathrm{gr}}$ Donnet détient désormais un poste clé pour influer sur la sélection des livres proposés chaque année. C'est aussi à cette époque qu'il demande à $\mathrm{M}^{\mathrm{gr}}$ de Montblanc de lui céder à mi-temps son grand vicaire. Celui-ci refuse, mais Dufêtre gardera néanmoins le titre honorifique de grand vicaire de Bordeaux.

Depuis quelques temps déjà, l'Euvre des bons livres de Bordeaux cherche à établir à Paris un bureau de correspondance pour l'examen des nouveaux livres. C'est chose faite en 1839, lorsque la Bibliothèque de Saint-Thomas d'Aquin, initialement réservée aux associés du Rosaire, se transforme en bibliothèque de bons livres, avec l'approbation de $\mathrm{M}^{\mathrm{gr}}$ de Quélen, puis devient à partir de 1842, le cœur de la rédaction de la Bibliographie catholique, revue créée pour rendre compte des travaux du comité d'examen chargé de l'analyse des nouvelles publications. L'introduction qui est donnée dans le premier tome de cette revue place explicitement cette initiative dans le prolongement de celle de Bordeaux, en fournissant un historique de l'Euvre et en reproduisant l'ordonnance

56. L'EEuvre lilloise, qui porte d'ailleurs le nom de "Bibliothèque de la société catholique ", dispose dès sa création de 2000 volumes pour un local qui peut en accueillir 15000. Cf. L'Ami de la religion et du roi, t. 46, p. 152. 
de 1825, où M ${ }^{\text {gr }}$ d'Aviau établissait l'organisation et le règlement de l'Euvre de Bordeaux. Désormais, les bibliothèques de bons livres, déjà invitées à s'affilier à l'archiconfrérie, se retrouvent fédérées autour du bureau d'examen de la Bibliographie catholique. L'entreprise permet d'uniformiser les méthodes et les critères présidant à la sélection des ouvrages. Elle répond ainsi efficacement à l'encyclique Mirari vos (1832) de Grégoire XVI qui soulignait le combat à mener pour assurer de « bonnes lectures », enfin soumises à une doctrine unique et infaillible.

Ce premier tome de la Bibliographie catholique, publié en 1842 à Paris chez Paul Renouard, n'est en fait qu'une seconde édition. La première est imprimée l'année précédente à Tours, chez Alfred Mame \& Cie57. Comment expliquer le fait, sinon par l'action influente dans ce projet parisien de $\mathrm{M}^{\mathrm{gr}}$ Donnet ${ }^{58} \ldots$ mais aussi du grand vicaire Dufêtre? Car il se trouve qu'en 1840, l'abbé Dufêtre fait oeuvre de prédication à Paris, précisément dans les paroisses de Saint-Thomas-d'Aquin et de Bonne Nouvelle ${ }^{59}$. Deux ans plus tôt, c'est la paroisse de Saint-Roch qui l'avait entendu. Ainsi nous retrouvons dans le parcours de Dufêtre trois des neuf paroisses parisiennes où figurent des bibliothèques de bons livres en 1842, d'après la cartographie qu'en donne Loïc Artiaga ${ }^{60}$. Fait révélateur, dès 1840, le Catalogue de la Bibliothèque de Saint-Thomas d'Aquin, encore classé par ordre alphabétique, signale à la lettre B la "Bibliothèque de la jeunesse chrétienne " de la maison Mame, aux côtés de la " Nouvelle Bibliothèque catholique " publiée par Lefort et de la "Bibliothèque instructive et amusante " des frères Gaume, fournissant pour chacun d'eux une liste de titres. En janvier 1844, c'est au tour de la Bibliographie catholique de citer, dans le cadre d'un " historique de l'Oeuvre des bons livres ", les excellentes collections publiées par la maison Mame, égales à celles de Lefort ${ }^{61}$... Mais la promotion de la maison Mame ne relève pas de la seule offensive médiatique. Elle doit aussi à la conception plus ciblée de ses collections.

En 1827, Lefort avait choisi de construire sa " Nouvelle Bibliothèque catholique " autour d'ouvrages accessibles, et donc à large diffusion, réunissant tout un panel de lecteurs. Le concept répondait alors parfaitement aux attentes de l'oeuvre bordelaise, dans la mesure où elle-même opérait ensuite, dans son catalogue à l'usage des bibliothécaires, un classement des titres assorti d'un lettrage par catégorie de lecteurs, s'assurant par là

57. Arch. dép. d'Indre-et-Loire, T845 : bulletin de dépôt légal du 21 août $1841 ;$ In- 8,2 f ${ }^{\circ}+$ couv., 1000 ex. (relevé effectué par François Fièvre - programme ANR « La maison Mame à Tours ").

58. L'abbé Bérault des Billiers, directeur de l'Euvre des bons livres de Saint-Thomas d'Aquin, explique, à propos de la création de cette revue mûrie pendant deux ans : "nous l'avons longuement méditée, nous l'avons communiquée à des personnes sages et amies du bien, [...] notamment celle du directeur de l'Euvre de Bordeaux, à laquelle nous attachons un grand prix ", Bibliographie catholique, 1842, t. 1, p. 11.

59. Crosnier, Augustin-Joseph, Vie de $M^{g r}$ Dufêtre..., op. cit., p. 69

60. Artiaga, Loïc, Des torrents de papier..., op. cit, p. 88.

61. Bibliographie catholique, t. 2, 1843-1844, p. 273. 
d'une orthopraxie du prêt et de la lecture. En 1841, lorsque Lefort publie un catalogue rétrospectif des 300 titres parus dans sa " Nouvelle Bibliothèque catholique $^{62}$ ", il ne peut, pour répondre aux besoins de sa clientèle, que reprendre le lettrage élaboré par l'Euvre bordelaise en l'adaptant au caractère "grand public " de sa collection (Annexe). Il prolonge ainsi un mode de promotion éditoriale très largement tributaire des pratiques liées aux bibliothèques paroissiales. Bien que les modalités de classement des ouvrages y soient susceptibles de variantes d'un diocèse à l'autre, la majorité d'entre elles adopte un classement par catégorie de lecteurs, parfois combiné à une organisation thématique ${ }^{63}$. Tel est le cas du Catalogue de la Bibliothèque de Saint-Thomas d'Aquin (1845) ${ }^{64}$ qui propose un classement en 27 sections. Les sections " Histoires de personnes, vies de saints " et "voyages " sont subdivisées, comme d'autres, en fonction du niveau d'instruction. D'un côté, les ouvrages " plus étendus, plus sérieux, plus appropriés aux lecteurs instruits "; de l'autre, ceux " à la portée des sections XIX, XXII, XXIII, XXV ". Si on se reporte à ces dernières, sont regroupés là enfants, ouvriers, domestiques et habitants des campagnes. La section XIX " enfants " ajoute à sa liste spécifique des renvois aux sections précédentes. Figurent les sections III : Instruction religieuse; IV : Piété; VII : Morale; VIII : Morale historique ou livre d'imagination contenant une instruction ou une moralité; IX : Histoire religieuse; XIII : Histoires de personnes, vies de saints ; XIV : Voyages ; XV : Sciences; XVI : Littérature. Ce classement permet d'apprécier la variété d'une bibliothèque chrétienne pour enfants, distincte de celle des jeunes gens et jeunes personnes (que l'on trouve en sections Xx et XXI). Enfin la section XXVII, qui clôt le catalogue et comme placée en appendice, est consacrée aux livres de prix des maisons d'éducation. Elle paraît témoigner des demandes croissantes associées à l'usage du catalogue : "Parmi les personnes vouées à l'éducation, beaucoup cherchent des renseignements sur les livres propres à être donnés en prix à leurs élèves. Nous avons tâché par [cette section] de leur venir en aide."

Ce phénomène s'explique par le développement de l'instruction publique. La loi Guizot (1833) favorise les écoles chrétiennes déjà implantées. Avec elles, c'est le marché du livre scolaire et du livre de prix qui se développe. Le phénomène s'accentue encore avec la loi Falloux (1850), une aubaine tout à la fois pour l'Église et pour les éditeurs catholiques engagés dans l'œuvre des bons livres. Mais, pour faire face à ce nouveau marché, le projet mené par le grand vicaire Dufêtre et les imprimeurs-libraires Mame se révèle sensiblement mieux construit que celui de la maison Lefort. Depuis longtemps déjà, Dominique Dufêtre travaille sur le terrain de l'éducation et son action s'étend sur de nombreuses congrégations enseignantes. À par-

62. Nouvelle Bibliothèque catholique : 300 volumes depuis janvier 1827 jusqu'à octobre 1841. Bib. mun. de Lille, 21839.

63. Il n'est pas impossible que ce classement soit emprunté à la tradition pédagogique des recueils d'exempla per status élaborés par les prédicateurs médiévaux.

64. Catalogue de la Bibliothèque de Saint-Thomas d'Aquin. Paris, Au bureau de la Bibliographie catholique, 1845. En ligne sur Gallica. 
tir de 1843, il soutient tout particulièrement les Sœurs de la charité, dont il dirige la maison mère à Nevers, mais il entretient également des liens étroits avec les frères des écoles chrétiennes et les clercs de Saint-Viateur, congrégation fondée par son ami Louis Querbes. Il encourage aussi les sœurs de Saint-Joseph, celles de la Sainte-Famille, celles de l'Enfant-Jésus, et d'autres. Beaucoup de ces congrégations essaimeront à l'étranger au cours du XIX ${ }^{\mathrm{e}}$ siècle, élargissant d'autant plus le marché du livre de classe et du livre de prix.

En s'orientant vers des collections spécifiquement conçues pour l'enfance et la jeunesse, les imprimeurs-libraires Mame héritent donc de l'autorité acquise par l'abbé Dufêtre dans le domaine de l'enseignement. Celle-ci est à ce point connue qu'en 1849, Alfred de Falloux, ministre de l'Instruction publique et des cultes, n'hésite pas à consulter $M^{\text {gr }}$ Dufêtre au sujet de son projet de loi ${ }^{65}$.

Ils bénéficient en outre de son expérience qui leur permet d'ajuster immédiatement leur production aux nouveaux usages du livre. Ainsi, entre 1836 et 1855, sept collections d'éducation voient le jour. Elles se partagent entre public familial et scolaire, enfants et adolescents. On y retrouve les genres que les bibliothèques de bons livres recommandent, notamment dans la Bibliothèque de la jeunesse chrétienne. Celle-ci avait débuté en 1836 avec des romans édifiants. Elle s'ouvre en moins de 3 ans à de nombreux domaines, parmi lesquels dominent néanmoins les sujets historiques. En 1841, la maison Mame obtient avec Poussielgue-Rusand, libraire à Paris un contrat d'exclusivité pour publier et vendre les manuels des Frères. La Bibliothèque de l'enfance chrétienne et la Bibliothèque des écoles chrétiennes viennent compléter ce marché spécifique par des publications propres à servir de livres de prix. Les collections pour l'enfance se restreignent, elles, aux vies de saints et aux historiettes morales. Cette relative pauvreté des genres est compensée par la déclinaison des titres : pas moins de 50 historiettes pour la Bibliothèque des petits enfants dès la première année $\mathrm{e}^{66}$. Contrairement aux autres collections, toutes approuvées par l'archevêque de Tours puis de Nevers, la Bibliothèque pieuse rassemble des titres aux approbations diverses. Ces textes proviennent sans doute d'un fonds commun aux bibliothèques de bons livres. Les vies de saints sont en effet un des premiers genres à avoir été publiés pour ces bibliothèques.

Si l'abbé Dufêtre se montre soucieux de trouver, pour ses collections, des auteurs capables de renouveler le répertoire d'Ancien Régime, il opère aussi par " récupération " des premiers titres composés pour les bibliothèques de bons livres. Ainsi en va-t-il du petit roman Mélanie et Lucette de $M^{\text {gr }}$ d'Aviau : Mame le publie pour la première fois en 1823, pour le dif-

65. Cf. Arch. dioc. de Nevers, 1D5, Liasse C, dossier 1 " Liberté de l'enseignement ", lettre 1.7 (Remerciements du ministre Falloux).

66. Voir Boulaire, Cécile, "Une logique de collections, de la bibliothèque de la jeunesse chrétienne à la bibliothèque des petits enfants " dans Mame : deux siècles d'édition pour la jeunesse, Rennes, PUR, à paraître. 
fuser sans doute auprès des dépôts affiliés à l'Euvre bordelaise. Lorsque le roman entre en 1837 dans la Bibliothèque de la jeunesse chrétienne, il connaît en fait sa neuvième édition. Mais le parcours de La Pieuse Paysanne ou la vie de Louise Deschamps est plus parlant encore. Ce grand classique des bibliothèques de bons livres raconte la vie d'abord dévoyée d'une paysanne du XVIII ${ }^{\mathrm{e}}$ siècle, puis sa conversion, en passant par les étapes de fille, d'épouse et de mère. Attribué à l'abbé Louis-Joseph de Baudry (1778$1854)^{67}$, ce roman est en fait le remaniement d'un récit plus ancien, Histoire de la vertueuse Portugaise ou le modèle des femmes chrétiennes, de l'abbé Maydieu, publié en 1779. Voici comment L'Ami de la Religion relate dans son numéro du 24 août 1822 (t. 33, p. 62-63) les étapes de son entrée dans les oeuvres de bons livres :

"La Vertueuse Portugaise n'était pas [...] exempte de défauts; l'auteur, après chaque trait d'histoire, interrompait son récit pour placer une ou deux pages de morale; il avait imaginé un épisode d'amour qui n'eut pas tous les suffrages, quelque louable que fut l'intention. Il y eut cependant une seconde édition, sans aucun changement, qui fut faite à Besançon en 1817. Deux prêtres zélés crurent pouvoir améliorer l'ouvrage. L'un d'eux, M. Phelipon, qui professe aujourd'hui la rhétorique avec succès au petit séminaire de Bordeaux, refondit la Vertueuse Portugaise, qui fut imprimée en $1820^{68}$ avec les approbations de M. l'archevêque de Bordeaux et de M. l'archevêque de Limoges. L'année suivante, on en fit une nouvelle édition, avec beaucoup de corrections et d'augmentations; elle diffère de la Vertueuse Portugaise au moins pour la moitié des articles. Un ecclésiastique, qui ne veut pas être connu, s'est chargé du travail [l'abbé de Baudry]. "

Mame édite La Pieuse paysanne en 1829. L'introduction souligne : "Ce petit ouvrage, qui a mérité l'approbation des prélats les plus respectables, n'est point écrit pour ceux qui chercheraient un style brillant et des expressions pompeuses. C'est aux habitants des campagnes qu'il est principalement destiné. " La chose est entendue. Pourtant nous retrouvons ce titre en 1857 dans la Bibliothèque des écoles chrétiennes, avec cette fois l'approbation de l'évêque de Nevers. Le roman a été ramené de 287 pages à 140 pages. Le souci de ne pas être trop long a présidé à cet élagage, à quoi s'ajoute celui de ne pas enflammer l'imagination de la jeunesse par quelques détails indiscrets, jusque-là tolérés à l'endroit des adultes.

67. Cet abbé sulpicien enseigna à partir de 1806 la philosophie et la théologie au séminaire Saint-Sulpice à Paris, avant de professer au séminaire de Limoges jusqu'en 1825, puis à Lyon. Il s'exila à Genève en 1830. D'origine savoyarde, il était aussi chanoine et vicaire général honoraire d'Annecy. Cf. BERTRAND, Louis, Bibliothèque sulpicienne, Paris, Alphonse Picard et fils, 1900, t. 2, p. 121-125. On peut se demander s'il ne fut pas un membre de l'AA d'Annecy et un relais pour les Amicizie dans la mesure où son texte connut en 1829 une édition en italien, chez l'éditeur lyonnais Rusand : La Contadina pia, ossia vita di Luigia Decampi. Son roman fut aussi édité au Québec dès 1823.

68. Cette première impression fut réalisée par Barbou Frères. Cf. Bib. mun. de Lyon, SJ V 582/18. L'imprimeur Barbou serait-il dès cette époque associé à l'EEuvre bordelaise pour que figure aussi l'approbation $\mathrm{M}^{\mathrm{gr}} \mathrm{d}$ 'Aviau? 
Dans tous les cas (créations ou adaptations), il s'agit de séduire le jeune public en empruntant à la fiction les recettes de son succès. À côté des traditionnelles histoires royales et des vies de saints, des berquinades en plein essor, s'ajoutent les récits de voyage liés aux Missions et les romans antiques qui célèbrent la foi de la première Église. Entre fiction et témoignage historique, le récit se plie toujours à une rhétorique apostolique globalement pensée. Du reste, l'abbé Dufêtre sait faire feu de tout bois. Il ira jusqu'à détrousser les "mauvaises lectures " que sont les " romans à la mode " pour mieux les plier à sa cause ${ }^{69}$.

Le développement exceptionnel de la maison Mame à Tours au $\mathrm{XIX}^{\mathrm{e}}$ siècle, et tout particulièrement sous le Second Empire, ne tient pas au seul dynamisme d'Alfred Mame et à ses ambitions industrielles, si souvent et légitimement soulignées. En replaçant cette réussite éditoriale dans le cadre de l'histoire de l'Église, cette étude vise à montrer que le développement de la maison tourangelle doit aussi à l'action du clergé. Sous la Restauration, l'Église de France s'est en effet lancée dans une opiniâtre politique de reconquête des âmes. Les missions se succèdent; elles contribuent à la création d'associations pieuses, œuvrant à l'ouverture d'écoles chrétiennes comme à celle de bibliothèques de bons livres. À Tours, le clergé diocésain participe lui aussi, entre 1817 et 1824, à cette double croisade. Celle-ci aboutit, en 1825, à la création d'une bibliothèque centrale de prêt, à laquelle sont rattachés des dépôts partiels, probablement dans les établissements scolaires et les paroisses. Amand Mame, qui s'est rapproché des cercles catholiques et légitimistes depuis 1818, est bien placé pour bénéficier des retombées économiques de ce nouvel apostolat par le livre. Et ce d'autant plus que le projet tourangeau s'inscrit dans une structure dont le réseau de diffusion dépasse le cadre de l'archevêché. En mettant au jour les liens amicaux et militants qui unissent les principaux acteurs tourangeaux à $\mathrm{M}^{\mathrm{gr}}$ d'Aviau, protecteur de l'oeuvre des bons livres de Bordeaux, cette étude témoigne de l'ampleur de l'offensive qui fut menée, s'appuyant sur un réseau de sociétés secrètes aux ramifications internationales. Les AAs françaises, tout comme les filiales de la Congrégation, propagent en France sous la Restauration le modèle d'apostolat par le livre promu par les Amicizie italiennes. Si, à Tours, les premiers acteurs de ce réseau disparaissent au début des années 1820, il revient au grand vicaire Dominique Dufêtre et au supérieur des Missionnaires de Saint-Martin, Ferdinand Donnet, formés au contact de ces sociétés secrètes, de poursuivre l'entreprise. En 1833, l'abbé Dufêtre convainc les héritiers d'Armand Mame, Ernest et Alfred Mame, de se lancer dans la publication de collections pour la jeunesse chrétienne dont il se réserve la direction éditoriale, les assurant de son concours le plus actif. C'est le début, pour la maison d'édition tourangelle, d'une straté-

69. Cf. LitAudon, Marie-Pierre, "Les dessous apologétiques d'un transfert littéraire : quand l'éditeur Mame adapte le premier pour la jeunesse française The Last Days of Pompeii de Bulwer Lytton ", Revue de Littérature Comparée (à paraître en 2011). 
gie de développement national, voire international, auquel Alfred Mame sut répondre en dotant son entreprise de structures modernes, adaptées à une production industrielle ${ }^{70}$. Le projet des abbés Dufêtre et Donnet apparaît, dans le contexte de l'époque, fort intelligemment pensé. Dufêtre semble avoir anticipé l'évolution des besoins liés au développement de l'instruction scolaire : les collections pour la jeunesse de l'éditeur Mame permettront de satisfaire les attentes nouvelles d'un public ciblé. Parallèlement, il a su gagner une autorité grandissante sur les congrégations enseignantes (principales prescriptrices).

Au milieu des années 1830, la maison Mame se trouve cependant sur un marché concurrentiel, où elle ne fait pas figure de leader. La maison Lefort à Lille, plus attachée aux sociétés parisiennes, avait lancé dès 1827 sa " Nouvelle Bibliothèque catholique " et elle bénéficiait du soutien médiatique des journaux ainsi que des catalogues des différentes Euvres de bons livres. Lorsque l'Euvre bordelaise est érigée en archiconfrérie en 1831, s'ouvre la perspective d'un catalogue unifié, diffusé jusque dans les Missions étrangères. Le défi pour l'entreprise Mame/Dufêtre est alors d'accéder aux postes décisionnels de l'Euvre bordelaise, mais aussi à ceux des journaux parisiens. Lorsque l'abbé Donnet est nommé à l'archevêché de Bordeaux en 1836, il obtient les moyens d'agir sur la première. Les années suivantes sont probablement l'occasion pour les abbés Dufêtre et Donnet de coordonner leurs actions pour disposer de relais dans les deuxièmes. En 1841 est créée la Bibliographie catholique, publication de référence en matière d'examen des nouveautés par les comités de bons livres. Les numéros de la première année sont imprimés sous les presses de la maison Alfred Mame \& $\mathrm{C}^{\mathrm{ie}}$. Hasard ou convergences d'intérêts, les publications de la maison Mame seront désormais mises à l'honneur. Pour confirmer cette dernière hypothèse, les correspondances des abbés Dufêtre et Donnet avec l'abbé Barrault des Billiers, fondateur et premier directeur de la Bibliographie catholique, mais aussi avec $\mathrm{M}^{\mathrm{gr}}$ de Quélen et $\mathrm{M}^{\mathrm{gr}}$ Affre, archevêques de Paris entre 1821 et 1848, pourraient apporter des éléments de réponse.

70. Cette restructuration de l'entreprise intervient à partir de 1845, lorsqu'Ernest Mame, moins entreprenant que son cousin, se retire des affaires pour entrer en politique. 


\section{Annexe - Catégories de lecteurs}

\begin{tabular}{|c|c|c|c|}
\hline \multicolumn{2}{|c|}{$\begin{array}{l}\text { Classement du catalogue de l'oeuvre borde- } \\
\text { laise (1834) }\end{array}$} & \multicolumn{2}{|c|}{$\begin{array}{l}\text { Classement du catalogue de la } \\
\text { "Nouvelle Bibliothèque catholique " de Lefort } \\
\text { (1841) }\end{array}$} \\
\hline A & $\begin{array}{l}\text { Enfants (filles et garçons qui n'ont pas } \\
\text { fait leur } 1^{\text {re }} \text { communion) }\end{array}$ & A & $\begin{array}{l}\text { Personnes dont l'instruction religieuse a } \\
\text { été tout à fait négligée }\end{array}$ \\
\hline B & $\begin{array}{l}\text { Jeunes gens (garçons qui ont fait leur } \\
1^{\text {re }} \text { communion et qui sont hors des } \\
\text { collèges, etc.; car ceux qui sont dans } \\
\text { les pensions, séminaires, etc. ont des } \\
\text { bibliothèques dans les maisons où ils } \\
\text { sont élevés) }\end{array}$ & B & $\begin{array}{l}\text { Personnes un peu plus instruites, qui ont } \\
\text { encore des préventions }\end{array}$ \\
\hline $\mathrm{C}$ & $\begin{array}{l}\text { Jeunes personnes hors des couvents, } \\
\text { etc. }\end{array}$ & $\mathrm{C}$ & Personnes qui ont lu des romans \\
\hline $\mathrm{D}$ & Gens mariés ou d'un âge mûr & $\mathrm{D}$ & Personnes pieuses \\
\hline $\mathrm{E}$ & $\begin{array}{l}\text { Personnes à qui ont veut inspirer } \\
\text { le goût des bons livres. (livres at- } \\
\text { trayants mais "innocents") }\end{array}$ & E & Militaires \\
\hline $\mathrm{F}$ & $\begin{array}{l}\text { Personnes instruites, capables de ju- } \\
\text { ger un livre savant ou bien écrit }\end{array}$ & $\mathrm{F}$ & Malades et personnes affligées \\
\hline $\mathrm{G}$ & Personnes peu instruites & G & $\begin{array}{l}\text { Personnes auxquelles on veut inspirer le } \\
\text { goût des bons livres }\end{array}$ \\
\hline $\mathrm{H}$ & $\begin{array}{l}\text { Gens d'une classe dépendante (do- } \\
\text { mestiques, journaliers, simples sol- } \\
\text { dats) }\end{array}$ & $\mathrm{H}$ & $\begin{array}{l}\text { Personnes sensibles aux beaux senti- } \\
\text { ments et qui ne pratiquent pas la religion }\end{array}$ \\
\hline I & & I & Jeunes gens \\
\hline $\mathrm{J}$ & $\begin{array}{l}\text { Lecteurs qui doutent de quelques } \\
\text { points de religion. Livres de contro- } \\
\text { verse }\end{array}$ & $\mathrm{J}$ & Enfants \\
\hline $\mathrm{K}$ & $\begin{array}{l}\text { Personnes pieuses qui aiment les } \\
\text { livres plein d'onction }\end{array}$ & $\mathrm{K}$ & $\begin{array}{l}\text { Ouvriers, domestiques et gens de la cam- } \\
\text { pagne }\end{array}$ \\
\hline $\mathrm{L}$ & $\begin{array}{l}\text { Personnes avancées dans la perfec- } \\
\text { tion, livres mystiques (ne sont prêtés } \\
\text { qu'avec réserve) }\end{array}$ & $\mathrm{L}$ & Personnes qui veulent se convertir \\
\hline M & Personnes affligées & M & Jeunes personnes \\
\hline $\mathrm{N}$ & $\begin{array}{l}\text { Scrupuleux (peu de livres leur sont } \\
\text { appropriés; leur refuser les livres } \\
\text { mystiques) }\end{array}$ & $\mathrm{N}$ & Personnes mariées \\
\hline $\mathrm{O}$ & $\begin{array}{l}\text { Ecclésiastiques (séminaristes en va- } \\
\text { cances, les autres ayant une biblio- } \\
\text { thèque) }\end{array}$ & $\mathrm{O}$ & $\begin{array}{l}\text { Ceux qui ont des relations avec les héré- } \\
\text { tiques }\end{array}$ \\
\hline $\mathrm{P}$ & Littérateurs & $\mathrm{P}$ & Hommes sans éducation \\
\hline $\mathrm{S}$ & Savants & $S$ & \\
\hline $\mathrm{Z}$ & & $\mathrm{Z}$ & $\begin{array}{l}\text { Ouvrages qui peuvent être mis entre les } \\
\text { mains de tout le monde }\end{array}$ \\
\hline
\end{tabular}




\section{RÉSUMÉ}

L'exceptionnel développement de la maison d'édition Mame à Tours, sous le Second Empire, a été maintes fois souligné par les historiens du livre et associé à la figure d'Alfred Mame, entrepreneur visionnaire et excellent organisateur. Cependant, en replaçant les étapes de cette réussite dans le cadre de l'histoire de l'Église, cette étude vise à montrer que la maison tourangelle n'aurait sans doute pas connu un tel essor si elle n'avait trouvé auprès de l'Église un puissant allié. La stratégie de la maison Mame se construit en effet dès la Restauration au contact du clergé ultramontain et légitimiste. Celui-ci est engagé dans une politique de reconquête des âmes par le livre mettant en jeu des sociétés plus ou moins secrètes aux ramifications internationales. De l'implication dans l'CEuvre des bons livres à la création de collections pour la jeunesse chrétienne, le parcours de la maison Mame, de la Restauration à l'avènement du Second Empire, témoigne de l'offensive menée.

\section{ABSTRACT}

The exceptional development of the Mame publishing house in Tours under the Second Empire has many a time been underlined by the historians of the book and associated with Alfred Mame, a visionary entrepreneur and an excellent organizer. However, by replacing the stages of this success story within the framework of the history of the Church, this study aims at showing that the Tours firm would probably not have known such a development if it had not found in the Church a powerful ally. Indeed, the Mame strategy, as early as the Restoration, was built in contact with the ultramontane and legitimist clergy. The latter was engaged in a politics of reconquest of souls through the book, involving more or less secret societies, with international ramifications. From its implication in the Good Books Society to the creation of series for the Christian Youth, the route of the Mame House, from the Restoration to the advent of the Second Empire testifies to the offensive then led. 